\title{
A Cross-Sectional Study of Isolates from Sputum Samples from Bacterial Pneumonia Patients in Trinidad
}

Nabeetha A. Nagalingam ${ }^{1}$, Abiodun A. Adesiyun ${ }^{2}$, William H. Swanston ${ }^{1}$ and Maria Bartholomew ${ }^{1}$
School of Medicine ${ }^{1}$ and School of Veterinary Science ${ }^{2}$, Faculty of Medicine, University of the West Indies, St. Augustine, Trinidad and Tobago

\begin{abstract}
We determined the frequency of Streptococcus pneumoniae, Haemophilus influenzae, Staphylococcus aureus, and Gram-negative enteric bacteria (GNEB) in pneumonia patients, determined the antibiograms of these pathogens, and investigated the relationship between pneumonia and selected risk factors. Sputum and demographic data were collected from 124 pneumonia patients. Sputum was cultured for S. aureus, GNEB, H. influenzae and S. pneumoniae. The disc diffusion method was used to determine resistance to eight antimicrobial agents. Among the 124 sputum samples, eight $(6.5 \%)$ were positive for $S$. aureus, $15(12.1 \%)$ for GNEB, two $(\mathbf{1 . 6 \%})$ for $S$. pneumoniae and one $(0.8 \%)$ for $H$. influenzae. Hospitals, gender, ethnicity, comorbidities and symptoms did not significantly $\left(p>0.05 ; \chi^{2}\right)$ affect the prevalence of these bacteria. GNEB infection was most prevalent $(47 \%)$ in patients over 70 years old. Gentamicin and levofloxacin were the most effective against these bacteria.

Key Words: Bacterial pneumonia, sputum, antibiogram.
\end{abstract}

Streptococcus pneumoniae is considered one of the most common causes of pneumonia [1,2]; it is the cause of up to $50 \%$ of pneumonia cases. In Spain, the prevalence ranges from $29-36 \%$ [3-5], which is comparable to the $35 \%$ found in France [6]. Pneumococcal infections are normally susceptible to penicillin and erythromycin; patients allergic to penicillin are prescribed erythromycin [7]. In the U.S.A., 25\% of isolates of S. pneumoniae exhibit low-level resistance to penicillin and about $15 \%$ show high-level resistance [7]; in that country, vancomycin is the drug of choice.

Staphylococcus aureus is cited as causing pneumonia in about $3 \%$ of patients with pneumonia worldwide $[3,6]$. In the U.S.A., prevalences of $0.5 \%$

Received on 18 February 2005; revised 11 June 2005.

Address for correspondence: Dr. Abiodun A. Adesiyun. School of Veterinary Science, Faculty of Medicine, The University of the West Indies, St. Augustine, Trinidad and Tobago (W.I.). E-mail: abiodunadesiyun@ hotmail.com Phone: 1-868-645-2640 ext4241 or 4237. Fax: 1-868-645-7428.

The Brazilian Journal of Infectious Diseases 2005;9(3):231-240 (C) 2005 by The Brazilian Journal of Infectious Diseases and Contexto Publishing. All rights reserved.
[8] and 5\% [9] have been documented, while in Japan the prevalence was $6.5 \%$ [10].

Haemophilus influenzae is responsible for $10 \%$ $38 \%$ of pneumonia cases $[3,11]$. In Spain, this bacterium was responsible for about $11 \%$ of pneumonia cases [3], while in the USA, studies have documented prevalences of $3 \%$ to $10.6 \%$ [8,9]. A relatively high prevalence of $H$. influenzae $(9.7 \%)$ was reported from Japan [10], compared with $1.8 \%$ in Switzerland [2].

Reported prevalences of community-acquired pneumonia (CAP) in the general population due to Gram-negative enteric bacteria (GNEB) vary from $0 \%$ to $9 \%$, with Pseudomonas aeruginosa accounting for $0 \%$ to $5 \%$ [3,12]. In Spain, the prevalence of GNEB was about $24 \%[3,4]$, while in the U.S.A. [8] and France [6], the prevalence was the same, 5\%. The GNEB have been associated with high mortality among pneumonia patients; because of this serious prognosis, risk groups should be treated carefully [3]. Pseudomonas aeruginosa plays a significant role, as it was implicated in $1.7 \%$ of pneumonia cases in a study done by Ruiz et al. [5]. The GNEB are most commonly E. coli, as reported by Pachon et al. [12], who found 
this species in about $22 \%$ of all pneumonia due to GNEB. Klebsiella spp. accounts for 1\%-5\%; Enterobacter spp., Serratia spp., Proteus spp., and Actinobacter spp. were also found [12].

Decisions concerning antibiotic therapy are greatly simplified if the pathogens are known [13]. As laboratory diagnoses are not always possible in time to treat the patients effectively, therapeutic guidelines have been put in place by the British and American Thoracic Societies [13]. It was suggested that $S$. pneumoniae should be treated with penicillin or amoxicillin, and during influenza epidemics, treatment against $S$. aureus should always be considered [13].

Bartlett et al. [13] suggested that hospitalized patients should be treated with second- and third-generation cephalosporins, with or without erythromycin. With the development of bacterial antibiotic resistance, as noted in Canada, where $H$. influenzae was found to be resistant to $\beta$-lactams [14] and S. pneumoniae resistant to penicillin and macrolides [1], the need for careful selection of antimicrobial therapy has become essential. Moreover, it is estimated that as many as $30 \%$ of avoidable deaths from pneumonia are due to incorrect selection of antimicrobial agents [15].

Approximately $90 \%$ of $S$. aureus isolates have plasmids that code for $\beta$-lactamase, an enzyme that degrades certain drugs, including many penicillins; but some penicillins, like methicillin, are resistant to $\beta$ lactamase [12]. However, some strains, such as methicillin-resistant strains of S. aureus (MRSA), have altered penicillin-binding proteins so that the penicillin cannot act. There are also rare strains called vancomycin - intermediate $S$. aureus (VISA) that have reduced sensitivity to vancomycin [7].

Pneumonia is ranked as the fifth leading cause of death in the world and the leading cause of infectious disease [12]. In Trinidad, it is the sixth leading cause of death and the second leading cause due to infectious disease, surpassed only by Acquired Immune Deficiency Syndrome (AIDS), according to the 1999 report from the Central Statistical Office (CSO) [16].

Our objective was to determine the frequency of selected bacteria in cases of pneumonia and to determine their susceptibility to common antimicrobial agents. This information will be useful in guiding empirical therapy of pneumonia in Trinidad.

\section{Materials and Methods}

The diagnosis of community-acquired pneumonia (CAP) requires the following to be present: a new pulmonary infiltrate seen on a chest radiograph that was taken within 24 hours of presentation; the confirmatory clinical finding includes at least one of the major criteria, which include, cough, sputum production, temperature $>37.8^{\circ} \mathrm{C}$; or at least two of the minor conditions, which include: pleuritic chest pains, dyspnea, altered mental status, pulmonary consolidation by physical examination, and white blood cell count of $>12,000$ cells $/ \mu \mathrm{L}$. The clinicians used these published criteria, and whenever they were met, they drew blood samples.

\section{Exclusion Criteria}

Patients in the following categories were excluded from the study: presence of tuberculosis, since this is highly contagious and therefore not allowed in the routine microbiological laboratory; presence of Human Immunodeficiency Virus (HIV) infection, since this condition leaves patients susceptible to infections that members of an otherwise healthy population would not be subjected to; children $<5$ yrs of age; admission from a nursing home or a hospital, so as to avoid the inclusion of possible nosocomial pneumonia cases.

\section{Types of Samples}

Sputum of pneumonia patients was sampled between February and October 2003. The sample population was 132 pneumonia cases from the four hospitals: Port of Spain General Hospitals (POSGH), Eric Williams Medical Sciences Complex (EWMSC), San Fernando General Hospitals (SFGH), and Sangre Grande Health Centre (SGHC). The samples were from patients between the ages of 5-70 years. POSGH is located in the northwest region of the island, 
EWMSC is in the north and SGHC is in the northeast. The northern part of the country is more urban than the central and southern parts; thus, a large population is served by these health facilities. SFGH is southwest and normally serves the south and central region inhabitants.

\section{Administration of Questionnaire}

Both a questionnaire and a letter of consent were provided for each participant in the study to complete. The questionnaire elicited demographic information (age, gender, ethnicity), risk factors for pneumonia (smoking, foreign travel) and experiences (current or in the past) of co-morbidities.

\section{$\underline{\text { Sample Collection and Transport }}$}

Samples of sputum were collected in sterile, screwtop containers. The expectorated sputum was taken by asking the patient to cough deeply into the container, followed by immediate screwing on of the cap. Samples were transported to the laboratory within two hours and processed immediately or refrigerated at $4^{\circ} \mathrm{C}$ as soon as possible [17].

\section{Isolation of Bacteria from Sputum}

A smear was prepared from the most purulent material in the sputum and the slide was left to dry in the biosafety cabinet and then heat-fixed. A gram stain was done using standard methods [18]. White blood cells (WBCs), squamous epithelial cells (SECs) and bacteria were investigated. Samples with more than 25 WBCs, and less than 10 SECs were deemed appropriate for culture [18]. Samples that did not meet these criteria were discarded. To isolate $S$. pneumoniae, the sputum samples were swabbed onto blood agar plates (BAP) and incubated at $35^{\circ} \mathrm{C}$ in $7 \%$ $\mathrm{CO}_{2}$ for $24 \mathrm{hrs}$. The plates were examined under magnification and colonies that showed alpha hemolysis and were button-shaped were subcultured onto BAP and incubated at $35^{\circ} \mathrm{C}$ and in $7 \% \mathrm{CO}_{2}$ overnight, as previously described [17]. For confirmation, suspect colonies were subcultured onto BAP and an optochin disc was placed onto the streaks; these were incubated at $35^{\circ} \mathrm{C}$ and $7 \% \mathrm{CO}_{2}$ for $24 \mathrm{hrs}$ to note zones of inhibition $=14 \mathrm{~mm}$. Staphylococcus aureus was isolated after the sputum sample was swabbed onto Baird Parker agar (BPA) and incubated at $35^{\circ} \mathrm{C}$ for $48 \mathrm{hrs}$. Colonies that were black or grayish-black and shiny were subcultured onto BPA and incubated at $35^{\circ} \mathrm{C}$ overnight. A representative colony was stained to identify clusters of Gram-positive cocci. Slide and tube coagulase tests were used to identify $S$. aureus [19]. Haemophilus influenzae was isolated by subculturing sputum swabs onto chocolate agar (CA) and incubating at $35^{\circ} \mathrm{C}$ and $7 \% \mathrm{CO}_{2}$ for $12-24 \mathrm{hrs}$. The plate was examined under magnification. Colonies that were white and glossy were subcultured onto CA and incubated at $35^{\circ} \mathrm{C}$ and $7 \% \mathrm{CO}_{2}$ overnight [17]. Confirmation was made by demonstrating satellitism with the $X$ and V test [17]. GNEB were isolated from the sputum sample by plating onto a MacConkey agar (MA) plate, incubated at $35^{\circ} \mathrm{C}$ for $24 \mathrm{hrs}$. Identification and confirmation of isolates was done using a battery of biochemical tests, as described by MacFaddin [19].

\section{Determination of Antibiograms of Isolates}

Antibiograms of the various pathogens were determined using the disc diffusion method [20]. The drugs and concentrations, chosen based on the frequency of prescription by doctors to pneumonia patients, were as follows: macrolide-erythromycin (E 15), cephalosporin-ceftazidime (CAZ 30), tetracycline-oxytetracycline (OT 30), sulphonamidesulphonamide (S3 300), aminoglycoside-gentamicin (CN 10), clindamycin-clindamycin (DA 2), 4quinolones-levofloxacin(LEV 5) and penicillin-oxacillin (OX 1). The zone sizes were interpreted for strains that were sensitive, intermediate and resistance, using published guidelines [20,21].

\section{$\underline{\text { Analysis of Data }}$}

Statistical analysis was done using $\chi^{2}$ tests with the Statistical Package for Social Sciences (SPSS) Version 9.0. 


\section{Results}

Prevalence of Selected Bacteria in Sputum

GNEB, excluding Pseudomonas spp., were the most prevalent microorganisms recovered, accounting for $15(12.1 \%)$ of the 124 sputum samples (Table 1). Among these, three were Aeromonas spp., three Moraxella spp., two Pasteurella spp., two Klebsiella spp., two E. coli, one Citrobacter spp., one Alcaligenes spp. and one Proteus spp. Haemophilus influenzae was isolated from only one patient, a woman of East Indian origin aged between 41 and 50 years. The two isolates of $S$. pneumoniae originated from two males of African origin, both smokers, aged from 21-30 and $41-50$ years, respectively.

Isolation of Bacteria in Pneumonia Patients by Source of Samples

A total of eight $S$. aureus isolates were recovered, four were isolated from 61 samples collected at San Fernando General Hospital, three were from the 28 samples at Sangre Grande Clinic, and the remaining one was from the 31 collected at Port of Spain General Hospital (Table 2). The differences in the prevalence of $S$. aureus across hospitals were not statistically significant ( $\mathrm{p}=0.647$ ), as was also found for both GNEB and Pseudomonas spp. The differences in the number of samples from males and females were not statistically significant for $S$. aureus, GNEB, or Pseudomonas spp. About half of the patients with GNEB were over 70 years old, accounting for seven of the 15 positive cases. The frequencies of isolation of GNEB by age of patients was not significantly different $(\mathrm{p}=0.12)$ since among the 15 cases, three were in the 51-60 age group, two were 61-70 years, and there was one in each of the categories; < 21years, 31-40 and 41-50.

A comparison of cases by age, gender and ethnicity was done to test whether these variables had an effect on the prevalence of the microbes; a significant difference was found for males $\left(\mathrm{p}=0.003 ; \chi^{2}\right)$ for GNEB. Amongst male patients, five of the 10 were in the over 70 age group, and only one from the 41 to 50 age group was East Indian, while all three in the 51-60 age group were African, and there was one in the 3140 age group of another ethnicity, showing that East Indian males over 70 years had significantly more GNEB.

Prevalence of Bacteria by Clinical Manifestation in $\underline{\text { Patients }}$

No significant differences were found between $S$. aureus positive patients who had abdominal pains, chest pains, chills, diarrhea, ear pains, fever, hoarseness, muscle pains, cough, vomiting, wheezing, confusion, loss of appetite or rash, compared to S. aureusinfected patients without these symptoms (Table 3). Likewise, among the GNEB-positive patients, there was no significant difference between the frequency of those with these various symptoms compared to that of those without such symptoms. There was a significant $(p=0.01)$ difference between GNEB patients with and without headaches; however, this did not relate to the bacterial infections, but may be due to other, untested, factors.

Prevalence of Bacteria in Pneumonia Patients by CoMorbidities

Comparisons among bacteria-positive patients revealed no significant differences for individuals with the underlying diseases: arthritis, asthma, chronic obstructive pulmonary disease (COPD), diabetes mellitus (DM), heart disease, hypertension, liver disease or renal insufficiency (Table 4).

\section{Antibiograms of Isolated Bacteria}

The S. pneumoniae and H. influenzae isolates were susceptible to all the antimicrobial agents tested. The highest frequency of resistance exhibited by S. aureus was to ceftazidime with five of eight isolates, while all were sensitive to gentamicin and erythromycin (Table 5). Two of the eight isolates were resistant to oxacillin. GNEB and Pseudomonas isolates exhibited a high 
Table 1. Prevalence of selected bacteria in 124 sputum samples from bacterial pneumonia patients

\begin{tabular}{lc}
\hline Bacteria & $\begin{array}{c}\text { Sputum } \\
\text { No. positive (\%) }\end{array}$ \\
\hline Streptococcus pneumoniae & $2(1.6)$ \\
Haemophilus influenzae & $1(0.8)$ \\
Staphylococcus aureus & $8(6.5)$ \\
GNEB & $15(12.1)$ \\
Pseudomonas spp. & $5(4.0)$ \\
\hline
\end{tabular}

GNEB: Gram-negative enteric bacteria; No.: number.

Table 2. Isolation of bacteria in pneumonia patients by source of samples

\begin{tabular}{|c|c|c|c|c|c|c|c|c|}
\hline & $\begin{array}{l}\text { S. aureus } \\
\text { No. }(\%) \\
\text { positive }\end{array}$ & $\mathbf{p}$ & $\begin{array}{c}G N E B \\
\text { No. }(\%) \\
\text { positive }\end{array}$ & p & $\begin{array}{c}\text { Pseudomonas } \\
\text { No. }(\%) \\
\text { positive }\end{array}$ & $\mathbf{p}$ & $\begin{array}{c}\text { S. pneumoniae } \\
\text { No. }(\%) \\
\text { positive }\end{array}$ & $\begin{array}{c}\text { H.influenzae } \\
\text { No. }(\%) \\
\text { positive }\end{array}$ \\
\hline \multicolumn{9}{|l|}{ Hospital } \\
\hline SFGH & $4 \quad(6.6)$ & 0.65 & $4 \quad(6.6)$ & 0.13 & $4(6.5)$ & 0.50 & $1(1.6)$ & $1(1.6)$ \\
\hline POSGH & $1 \quad(3.2)$ & & $7(22.6)$ & & $1(3.2)$ & & $1(3.2)$ & $0(0.0)$ \\
\hline SGHC & $3(10.7)$ & & $4(14.3)$ & & $0(0.0)$ & & $0(0.0)$ & $0(0.0)$ \\
\hline EWMSC & $0 \quad(0.0)$ & & $0 \quad(0.0)$ & & $0(0.0)$ & & $0(0.0)$ & $0(0.0)$ \\
\hline \multicolumn{9}{|l|}{ Gender } \\
\hline Male & $5 \quad(7.9)$ & 0.25 & $10 \quad(5.9)$ & & $3(4.7)$ & & $2(3.2)$ & $0(0.0)$ \\
\hline Female & 3 (4.9) & & $5 \quad(8.2)$ & & $2(3.3)$ & & $0(0.0)$ & $1(1.6)$ \\
\hline \multicolumn{9}{|l|}{ Age } \\
\hline$<21$ & $0 \quad(0.0)$ & 0.47 & $1 \quad(7.1)$ & 0.12 & $1(7.1)$ & 0.74 & $0(0.0)$ & $0(0.0)$ \\
\hline $21-30$ & $1 \quad(8.3)$ & & $\begin{array}{ll}0 & (0.0)\end{array}$ & & $1(8.3)$ & & $1(8.3)$ & $0(0.0)$ \\
\hline $31-40$ & $1(12.5)$ & & $1(12.5)$ & & $0(0.0)$ & & $0(0.0)$ & $0(0.0)$ \\
\hline $41-50$ & $1 \quad(5.3)$ & & $1 \quad(5.3)$ & & $0(0.0)$ & & $1(5.3)$ & $1(5.3)$ \\
\hline $51-60$ & $4(13.8)$ & & $3(10.3)$ & & $2(6.9)$ & & $0(0.0)$ & $0(0.0)$ \\
\hline $61-70$ & $0 \quad(0.0)$ & & $2(10.5)$ & & $0(0.0)$ & & $0(0.0)$ & $0(0.0)$ \\
\hline$>70$ & $1 \quad(4.3)$ & & $7(30.4)$ & & $1(4.2)$ & & $0(0.0)$ & $0(0.0)$ \\
\hline \multicolumn{9}{|l|}{ Ethnicity } \\
\hline East Indian & $2 \quad(3.2)$ & 0.31 & 7 (11.1) & 0.26 & $3(4.8)$ & 0.53 & $0(0.0)$ & $1(1.6)$ \\
\hline African & $4(11.8)$ & & 3 (7.9) & & $2(5.3)$ & & $2(5.3)$ & $0(0.0)$ \\
\hline Other & $2 \quad(8.7)$ & & $5(21.7)$ & & $0(0.0)$ & & $0(0.0)$ & $0(0.0)$ \\
\hline
\end{tabular}

Hospitals: Port of Spain General Hospitals (POSGH), Eric Williams Medical Sciences Complex (EWMSC), San Fernando General Hospitals (SFGH), and Sangre Grande Health Centre (SGHC). 
Table 3. Prevalence of bacteria by clinical manifestation in bacterial pneumonia patients

\begin{tabular}{lcccccc}
\hline Signs/symptoms & $\begin{array}{c}\text { S. aureus } \\
\text { No. }(\%) \\
\text { positive }\end{array}$ & $\mathbf{p}^{\mathbf{a}}$ & $\begin{array}{c}\text { GNEB } \\
\text { No. (\%) } \\
\text { positive }\end{array}$ & $\mathbf{p}^{\mathbf{a}}$ & $\begin{array}{c}\text { Pseudomonas spp. } \\
\text { No. (\%) } \\
\text { positive }\end{array}$ & $\mathbf{p}^{\mathbf{a}}$ \\
\hline Abdominal pains & $3(6.8)$ & 0.53 & $5(8.9)$ & 0.14 & $1(2.3)$ & 0.28 \\
Chest pains & $6(8.7)$ & 0.22 & $8(10.1)$ & 0.25 & $5(6.3)$ & 0.29 \\
Chills & $3(4.2)$ & 0.26 & $7(9.9)$ & 0.28 & $3(4.2)$ & 0.48 \\
Diarrhea & $2(6.5)$ & 0.59 & $2(6.5)$ & 0.23 & $1(3.2)$ & 0.52 \\
Difficulty breathing & $4(5.1)$ & 0.43 & $8(10.3)$ & 0.30 & $5(6.3)$ & 0.26 \\
Earpain & $1(4.2)$ & 0.56 & $2(8.3)$ & 0.42 & $0(0.0)$ & 0.25 \\
Fever & $4(9.5)$ & 0.34 & $10(12.2)$ & 0.57 & $3(3.6)$ & 0.25 \\
Headache & $5(6.6)$ & 0.54 & $5(6.6)$ & 0.01 & $4(5.2)$ & 0.64 \\
Hoarseness & $3(5.4)$ & 0.66 & $5(8.9)$ & 0.17 & $4(7.1)$ & 0.29 \\
Muscle pains & $6(8.2)$ & 0.13 & $8(11.0)$ & 0.44 & $4(5.4)$ & 0.58 \\
Productive cough & $5(5.4)$ & 0.43 & $12(12.9)$ & 0.31 & $5(5.3)$ & 0.63 \\
Vomiting/nausea & $3(5.5)$ & 0.60 & $8(14.8)$ & 0.24 & $2(3.7)$ & 0.45 \\
Wheezing & $3(5.5)$ & 0.58 & $9(16.4)$ & 0.10 & $2(3.6)$ & 0.42 \\
Mental confusion & $0(0.0)$ & 0.20 & $1(4.3)$ & 0.19 & $1(4.3)$ & 0.69 \\
Rash & $1(10.0)$ & 0.43 & $1(10.0)$ & 0.64 & $0(0.0)$ & 0.58 \\
Loss of appetite & $5(6.3)$ & 0.59 & $11(13.9)$ & 0.19 & $5(6.3)$ & 0.28 \\
\hline
\end{tabular}

${ }^{\mathrm{a}}$ values $<0.05$ are considered statistically significant.

Table 4. Prevalence of bacteria in pneumonia patients by co-morbidities

No. (\%) of samples positive for:

\begin{tabular}{lllllrr}
\cline { 2 - 7 } & $\begin{array}{c}\text { S. aureus } \\
\text { No. }(\%) \\
\text { positive }\end{array}$ & $\mathbf{p}$ & $\begin{array}{c}\text { GNEB } \\
\text { No. (\%) } \\
\text { positive }\end{array}$ & $\mathbf{p}$ & $\begin{array}{c}\text { Pseudomonas spp. } \\
\text { No. (\%) } \\
\text { positive }\end{array}$ & p \\
\hline Asthma & $0(0.0)$ & 0.38 & $2(13.3)$ & 0.50 & $0(0.0)$ & 0.53 \\
COPD & $0(0.0)$ & 0.89 & $0(0.0)$ & 0.791 & $0(0.0)$ & 0.91 \\
Diabetes mellitus & $2(9.5)$ & 0.33 & $2(9.5)$ & 0.58 & $1(4.5)$ & 0.74 \\
Heart disease & $2(7.4)$ & 0.46 & $1(3.7)$ & 0.15 & $0(0.0)$ & 0.22 \\
Hypertension & $1(3.8)$ & 0.56 & $4(15.4)$ & 0.31 & $0(0.0)$ & 0.24 \\
Liver disease & $0(0.0)$ & 0.80 & $0(0.0)$ & 0.62 & $0(0.0)$ & 0.83 \\
Renal insufficiency & $0(0.0)$ & 0.63 & $0(0.0)$ & 0.38 & $1(12.5)$ & 0.32 \\
\hline
\end{tabular}

a $\mathrm{P}$ values $<0.05$ are considered statistically significant. 
Table 5. Antibiograms of isolated bacteria

\begin{tabular}{|c|c|c|c|c|}
\hline \multirow[t]{2}{*}{ Antimicrobial agents } & \multirow[t]{2}{*}{ Disc concentration (ig) } & \multicolumn{3}{|c|}{ No. of isolates (\%) } \\
\hline & & Susceptible & Intermediate & Resistant \\
\hline \multicolumn{5}{|l|}{ S. aureus } \\
\hline $\mathrm{CAZ}^{\mathrm{a}}$ & 30 & $1 \quad(12.5)$ & $2(25.0)$ & $5(62.5)$ \\
\hline $\mathrm{CN}$ & 10 & $8(100.0)$ & $0 \quad(0.0)$ & $0 \quad(0.0)$ \\
\hline DA & 2 & $6 \quad(75.0)$ & $0 \quad(0.0)$ & $2(25.0)$ \\
\hline $\mathrm{E}$ & 15 & $2(25.0)$ & $2(25.0)$ & $4(50.0)$ \\
\hline LEV & 5 & $8(100.0)$ & $0 \quad(0.0)$ & $0 \quad(0.0)$ \\
\hline $\mathrm{OX}$ & 1 & $6 \quad(75.0)$ & $0 \quad(0.0)$ & $2(25.0)$ \\
\hline OT & 30 & $7 \quad(87.5)$ & $0 \quad(0.0)$ & $1(12.5)$ \\
\hline S3 & 300 & $7 \quad(87.5)$ & $0 \quad(0.0)$ & $1(12.5)$ \\
\hline \multicolumn{5}{|c|}{ GNEB and Pseudomonas spp. } \\
\hline CAZ & 30 & $18 \quad(69.2)$ & $2(7.7)$ & $6(23.1)$ \\
\hline $\mathrm{CN}$ & 10 & $26 \quad(100)$ & $(0)$ & $0 \quad(0)$ \\
\hline $\mathrm{DA}$ & 2 & $(0)$ & $(0)$ & $26(100)$ \\
\hline $\mathrm{E}$ & 15 & $(7.7)$ & $3(11.5)$ & $22(84.6)$ \\
\hline LEV & 5 & (100) & $(0)$ & (0) \\
\hline $\mathrm{OX}$ & 1 & $26 \quad(100)$ & $(0)$ & (0) \\
\hline OT & 30 & $14 \quad(53.8)$ & $4(15.4)$ & $8(30.8)$ \\
\hline S3 & 300 & $23 \quad(88.5)$ & $2(7.7)$ & $1 \quad(3.8)$ \\
\hline
\end{tabular}

${ }^{1}$ CAZ: ceftazidime, CN: gentamicin, DA: clindamycin, E: erythromycin, LEV: levofloxacin, OT: oxytetracycline, OX: oxacillin, S: sulphonamide.

frequency of resistance to clindamycin, oxacillin and erythromycin with 26, 26 and 22 of 26 isolates, respectively. All isolates were sensitive to gentamicin and levofloxacin.

\section{Discussion}

Sputum was cultured instead of blood, since only about $6 \%$ of blood cultures yield significant organisms, as observed by Campbell et al. in 2003 [22], compared with $55 \%$ of valid sputum samples collected in a study by Ewig et al. in 2002 [23]. Lung aspirates and tissue were difficult to obtain, hence were not the samples of choice.

The finding of two $S$. pneumoniae isolates, representing $1.6 \%$ ( 2 of 124 ) of the bacteria detected from the sputum of the pneumonia cases, is considerably less frequent than in some published studies where other methods of detection, including serology, were used; prevalences of from $20-60 \%$ were reported [5-7,13]. However, other studies have revealed comparable values, as was found in Ohio in the U.S.A., where an isolation rate of $5.5 \%$ was found amongst 154 patients [8]. In addition, Bochud et al. [2] isolated this pathogen from $3.5 \%$ of the sputum samples tested in Switzerland. The comparatively lower detection rate for $S$. pneumoniae may be a reflection of the insensitivity of sputum Gram staining and culture for this organism, as reported by Martson et al. [8], who found that only $44.4 \%$ of the patients with $S$. pneumonia infection had positive sputum cultures. Bochud et al. [2] noted that several of the studies quoting high $S$. pneumoniae detection had employed at least 
three diagnostic methods to find this bacterium, based on sputum, blood and pneumococcal antigens. Caution must therefore be exercised when comparing prevalence data on $S$. pneumoniae.

The rather low prevalence $(0.8 \%)$ of $H$. influenzae found in pneumonia cases in our study is similar to published reports, such as $0.4 \%$ by Martson et al. [8] from the U.S.A., and $0 \%$ by Bochud et al. [2] in Switzerland. These findings suggest that $H$. influenzae may not be an important etiological agent for pneumonia in these areas. The widespread low prevalence of the pathogen may be a consequence of a worldwide vaccination program against $H$. influenzae, serotype $\mathrm{B}$, launched after it was recognized how severe infections can be, especially in children [24]. Prior to this vaccination campaign, the incidence of disease due to $H$. influenzae was high, particularly in children developing pneumonia [24]. To generalize for all developing regions before vaccination, the Gambian estimate of the incidence of pneumonia (acute lower respiratory infection) from the early 1990s was 300 episodes per year per 100,000 in children younger than five years. This estimate may be considered conservative if up to $25 \%$ of cases of severe pneumonia of childhood are caused by $H$. inflenzae, as reported by Peltola et al. [24]. The fact that Trinidad and Tobago also participated in the vaccination program, although not as aggressively until 2000, may explain, in part, the low isolation rate of $H$. influenzae, as was also found in other developing countries [24].

The prevalence of $6.5 \%$ found for $S$. aureus in sputum samples is similar to the $3.8 \%$ found by Moine et al. [6] in France. On the other hand, a lower prevalence $(0.4 \%)$ was reportedly found in the U.S.A [8]. It is expected that $S$. aureus would contribute to pneumonia since it forms part of the natural oropharyngeal flora in some individuals [7]. Any breakdown in the immunity of these individuals can leave them open to invasion of the lung by this microbe. Staphylococcus aureus pneumonia has been reported to develop after a person inhales the organism or may result secondary to hematogenous seeding of the lungs in patients with bacteremia [7]. Inhalation $S$. aureus pneumonia has been reported during influenza epidemics, and it can lead to inflammation of the lung tissue, causing pneumonia [25].

The prevalence of $12.1 \%$ that we found for GNEB is comparable to findings reported by the American Thoracic Society of 3-10\% (13). Ruiz et al. [5] found two cases of Moraxella spp. among 182 patients with pneumonia in Spain. According to Mandell et al. [26], Moraxella is not a common cause of pneumonia, as reflected by the finding of only three cases among 124 sputum samples. Martson et al. [8] found that $0.1 \%$ of samples tested in the U.S.A. were positive for Klebsiella spp., a prevalence considerably lower than the $1.6 \%$ found in our study and with the report of Ruiz et al. [5] in Spain, where the organism was isolated from $1 \%$ of the patients with pneumonia. Martson et al. [8] also found that $0.6 \%$ of the samples tested positive for E. coli. in the U.S.A., while Ruiz et al. [5] found $2 \%$ and $1 \%$ positive, respectively, for E. coli and Proteus spp. The findings in these studies agree with the prevalence of $1.0 \%$ E. coli found in our study. The $4.0 \%$ prevalence of Pseudomonas detected in our study is similar to the $3.6 \%$ (20 of 559) found by Arancibia et al. [3] in sputum samples for patients in Spain, but Ruiz et al. [5] reported as high as 7.7\%, also in Spain. Arancibia et al. [3] postulate that the high frequency of recovery of GNEB may be due to structural lung disease, a factor that was not conclusively tested for in our study. These authors further explained that GNEB and Pseudomonas easily colonize the tracheobronchial tree whenever there is damage to the respiratory epithelium.

We found that among the patients with GNEB, $46.7 \%$ occurred in individuals in the over 70 -years age group. The fact that most of the GNEB were recovered from the over 70-age bracket was not unexpected, since El-Solh et al. [27] in the U.S.A. reported that $20 \%$ of all bacteria detected from pneumonia cases in the elderly are GNEB. According to Arancibia et al. [3], Gram-negative bacteria are well known to colonize the airways, being increasingly frequent with advanced age, but they found no significant differences that would indicate that age is a predisposing factor to infection. In addition, $P$. aeroginosa has been found to colonize the airways of the elderly [3]. These findings can be explained, in part, by the lowered immune response 
often associated with aging, which allows naturallyoccurring flora from the upper-respiratory tract, which may include Gram-negative bacteria like E. coli and even Pseudomonas, since these organisms can be very aggressive [3], to invade the lower-respiratory tract. Also, the elderly tend to be attended at hospitals more often than younger people, since they are more likely to suffer from co-morbidities that require treatment, a fact also seen in our study; hence they are more frequently exposed to GNEB, which, like MRSA, are present in the health care setting [3].

The finding of no significant differences in the bacteria isolated among the hospitals was expected, since Trinidad is relatively small and the population is not isolated and thereby would not show geographical differences. In fact, it is a common practice to send patients from one hospital to another, whenever there are demands on space and equipment.

Although the prevalence, by gender of patients, of each of the bacteria did not differ significantly, overall the prevalence of infection was higher in male than in female patients. A similar finding was reported by Miyashita et al. [10] in Japan, where infection by $S$. pneumoniae was found to be higher in males $(\mathrm{n}=$ 38 ) than in females $(n=20)$. Kaplan et al. [14] found that men are more likely to be hospitalized with CAP than are woman. Further studies are needed to assess, not only biological factors, but also access to health care, since biological response to infection, sex bias in hospital admission and patterns of health care delivery may be factors that lead to more males being admitted with CAP [9].

It was of interest to find that the prevalences of the various bacteria were not significantly different amongst the ethnic groups in Trinidad. Martson et al. [8] had reported that people of African decent are more susceptible to pneumonia than Caucasians. They indicated that such differences may have be due to a socio-economic status, but then went on to admit that these findings are incompletely understood.

The finding of no significant relationship between frequency of bacterial isolates and existence of underlying disease in patients may be explained, in part, by the findings of Riquelme et al. [4], who reported that not all the signs and symptoms are represented in all the cases of pneumonia. This may be due to interactions with underlying diseases. These authors reported that cough, fever and dyspnea are absent in $56 \%$ of pneumonia cases, and $96 \%$ of CAP patients had at least one respiratory symptom. Consequently, studies attempting to elucidate etiology from clinical parameters have poor results [4].

The finding that treatment of GNEB showed a good response, with $100 \%$ susceptibility to gentamicin and levofloxacin, was not unexpected, considering that these drugs target GNEB. The $100 \%$ and $84.6 \%$ prevalence of resistance found against clindamycin and erythromycin, respectively are also not surprising since these drugs target Gram-positive cocci.

The detection of MRSA is an indication that there is a threat, with rapid development of resistance to firstline regimens of treatments, as reported by Hoban et al. [14]. Orrett [28] had earlier reported the existence of MRSA in Trinidad. Staphylococcus aureus has shown great adaptability against antibiotic therapy, with penicillin resistance, and now methicillin resistance, developing.

The susceptibility of both Gram-positive and Gramnegative microbes to levofloxacin, a fluroquinolone, indicates that these bacteria have not yet developed resistance to this new drug. The evidence that the fluoroquinolones are effective against these respiratory pathogens may make these drugs an alternative to the more widely-used macrolides and cephalosporins in the Trinidadian population, in order to avoid the development of drug resistance. Also, the availability of drugs as oncedaily treatments and the feasibility of intravenous-to-oral sequential therapy are advantageous [26]. However, serious side effects to this drug, including dizziness, hypoglycemia, phototoxicity and hepatotoxicity are causes of concern for its use [29]. Since gentamicin and levofloxacin both work against Gram-positive and Gram-negative bacteria, they are good choices for broad-spectrum therapy. Often, this therapy is used when the laboratory findings are inconclusive or treatment is needed immediately, before laboratory diagnoses are available, forcing clinicians to implement "blind therapy". 


\section{Acknowledgements}

The authors extend their sincere thanks to the medical staff of the San Fernando and Port of Spain General Hospitals, as well as the Sangre Grande Health Clinic and the Eric Williams Medical Sciences Complex staff who assisted in patient identification and sample collection. This study would not have been possible without the financial support of the University of the West Indies Campus Research Committee.

\section{Reference}

1. Davidson R.J., Canadian Bacterial Surveillance Network, Low D.E. A cross-Canada surveillance of antimicrobial resistance in respiratory pathogens. Can J Infect Dis 1999; 10:128-33.

2. Bochud P.Y., Moser F., Erard P., et al. Community-acquired pneumonia. A prospective outpatient study. Medicine 2001;80(2):75-87.

3. Arancibia F., Bauer T.T., Ewig S., et al. Community-acquired pneumonia due to Gram-negative bacteria and Pseudomonas aeruginosa. Arch Inter Med 2002;162:1849-58.

4. Riquelme R., Torres A., El-Ebiary M., et al. Communityacquired pneumonia in the elderly- A multivariate analysis of risk and prognostic factors. Am J Respir Crit Care Med 1996; $154: 1450-5$.

5. Ruiz M., Ewig S., Marcos A.M., et al. Etiology of Community - Acquired pneumonia: Impact of age, comorbidity and severity. Am J Respir Crit Care Med 1999; 160:397-405.

6. Moine P., Vercken J.B., Chevret S., et al. Clinical investigation in critical care- Severe communityacquired pneumonia: Etiology, epidemiology, and prognosis factors. Chest 1994;105:1487-95.

7. Levinson W., Jawetz E. Medical microbiology \& immunology. Seventh edition. Appleton and Lange, New York, 1998.

8. Martson B.J., Plouffe J.F., File T.J., et al. Incidence of community-acquired pneumonia requiring hospitalization. Arch Intern Med 1997;157:1709-18.

9. Kaplan V., Angus D.C., Griffin M.F. et al. Hospitalized community-acquired pneumonia in the elderly. Age- and sex-related patterns of care and outcome in the United States. Am J Respir Crit Care Med 2002;165:766-72.

10. Miyashita N., Fukano H., Okimoto N., et al. Clinical presentation of community-acquired Chlamydia pneumoniae pneumonia in adults. Chest 2002;121:1776-81.

11. Kyprianou A., Hall C.S., Shah R., Fein A.M. The challenge of non resolving pneumonia. Postgrad Med 2003;113(1):79-92.
12. Pachon J., Prados M.D., Capote F., et al. Severe communityacquired pneumonia- Etiology, prognosis and treatment. Am Rev Respir Dis 1990;142:369-73.

13. Bartlett J.G., Mundy L.M. Current concept- Communityacquired pneumonia. NEngl J Med 1995;333(24):1618-24.

14. Hoban D., Karlowsky J., Zhanel G. Cross Canada surveillance susceptibility testing of Haemophilus influenzae with $\beta$-lactams, trimethoprim/ sulfamethoxazole, and clarithromycin. In: Program and abstracts of the $38^{\text {th }}$ International Conference on Antimicrobial agents and chemotherapy by the American Society for Microbiology; Washington DC, 1998.

15. Dubois R.W., Brooks R.H. Preventable deaths: who, how often and why. Ann of Intern Med 1988;109:582-9.

16. Republic of Trinidad and Tobago. Central Statistical OfficeBirth, Death and Population. Port of Spain: Trinidad, 1999.

17. Pan America health organization regional systems for vaccines (SIREVA). Streptococcus pneumoniae and Haemophilus influenzae identification and susceptibility testing. Proceedings of the Insituto Nacional de Saluda; Nov 23- Dec 1; Santa Fe de Bogota, 1998.

18. Clinical Microbiology Procedures Handbook. Chapter 8. American Society for Microbiology. USA: Isenberg HD.

19. MacFaddin J.F. Biochemical tests for identification of medical bacteria. Third edition. Lippincott, Williams and Wilkins, Philadelphia, U.S.A. 2000.

20. NCCLS. Performance standards for antimicrobial disk and dilution susceptibility tests for bacteria isolated from animals; approved standards. 2nd ed. M31-A2.;22 (6).

21. BBL Sensi-Disc Antimicrobial Susceptibility Test Discs [package insert]. Cockeysville, MD: Becton Dickinson and Co. 1993.

22. Campbell S.G., Marrie T.J., Nnstey R. et al. The Contribution of blood cultures to the clinical management of adult patients admitted to the hospital with communityacquired pneumonia. Chest 2003; 123:1142-50.

23. Ewig S., Schlochtermeier M., Göke N., Neiderman M.S. Applying sputum as a diagnostic tool in pneumonia. Chest 2002; $121: 1486-92$.

24. Peltola H. Worldwide Haemophilus influenzae Type b Disease at the Beginning of the 21st Century: Global analysis of the disease burden 25 years after the use of the polysaccharide vaccine and a decade after the advent of conjugates. Clin Microb Rev 2000;13(2):302-17.

25. Fakih M.G., Saravolatz L. Treatment of Staphylococcus aureus pneumonia. Curr Treat Opt Infect Dis 2003;5:137-42.

26. Mandell L.A., Marrie T.J., Grossman R.F. et al. Canadian guidelines for the initial management of communityacquired pneumonia: An evidence-based update by the Canadian Infectious Diseases Society and the Canadian Thoracic Society. Clin Infect Dis 2000;31:383-421.

27. El-Sohl A.A., Sikka P., Ramadan F., Davis J. Etiology of severe pneumonia in the very elderly. Am J Respir Crit Car Med 2001; 163 (3 Pt 1):645-51.

28. Orrett F.A. Methicillin resistance among Trinidadian isolates of community and hospital strains of Staphylococcus aureus and their patterns of resistance to non-beta-lactam antibiotics. Jpn J Infect Dis 1999; 52: 238-241. 